JOURNAL OF THE

CHUNGCHEONG MATHEMATICAL SOCIETY

Volume 27, No. 2, May 2014

http://dx.doi.org/10.14403/jcms.2014.27.2.243

\title{
ON CHARACTERIZATIONS OF PARETO AND WEIBULL DISTRIBUTIONS BY CONSIDERING CONDITIONAL EXPECTATIONS OF UPPER RECORD VALUES
}

\author{
Hyun-Woo Jin* And Min-Young LeE**
}

\begin{abstract}
Let $\left\{X_{n}, n \geq 1\right\}$ be a sequence of i.i.d. random variables with absolutely continuous cumulative distribution function(cdf) $F(x)$ and the corresponding probability density function(pdf) $f(x)$. In this paper, we give characterizations of Pareto and Weibull distribution by considering conditional expectations of record values.
\end{abstract}

\section{Introduction}

Let $\left\{X_{n}, n \geq 1\right\}$ be a sequence of independent identically distributed (i.i.d.) random variables with cumulative distribution function(cdf) $F(x)$ and probability density function (pdf) $f(x)$. Let $Y_{n}=\max \left\{X_{1}, X_{2}\right.$, $\left.\cdots, X_{n}\right\}$ for $n \geq 1$. We say $X_{j}$ is an upper record value of this sequence, if $Y_{j}>(<) Y_{j-1}$ for $j>1$. The indices at which the upper record values occur are given by the record times $\{U(n), n \geq 1\}$, where $U(n)=\min \left\{j \mid j>U(n-1), X_{j}>X_{U(n-1)}, n \geq 2\right\}$ with $U(1)=1$. We assume that all upper record values $X_{U(i)}$ for $i \geq 1$ occur at a sequence $\left\{X_{n}, n \geq 1\right\}$ of i.i.d. random variables.

Lee(2003) showed that $X \in P A R(\theta)$ if and only if $(\theta+1)^{i} E\left[X_{U(n+i)} \mid\right.$ $\left.X_{U(m)}=y\right]=\theta^{i} E\left[X_{U(n)} \mid X_{U(m)}=y\right]$ for $i=1,2,3, n \geq m+1$. Also, Dembinska \& Wesolowski(2000) obtained characterizations of the exponential, Pareto, and power distributions by considering this problem with $E\left(R_{m+k} \mid R_{m}\right)=a R_{m}+b$. Further, Ahsanullah and Shakil(2012)

Received January 24, 2014; Accepted April 07, 2014.

2010 Mathematics Subject Classification: Primary 62E10; Secondary 62G30, $62 \mathrm{H} 05,62 \mathrm{H} 10$.

Key words and phrases: absolutely continuous, characterizations, independent and identically distributed, record values, conditional expectations, Weibull distribution.

Correspondence should be addressed to Min-Young Lee, leemy@dankook.ac.kr. 
proved that $X \in P A R(\beta)$ if and only if $(\delta-k) E\left[X_{U(n+1)}^{k} \mid X_{U(m)}=\right.$ $y]=\delta E\left[X_{U(n)}^{k} \mid X_{U(m)}=y\right], m \geq n+1$.

In this paper, we give the characterizations of Pareto and Weibull distributions by considering conditional expectations of record values.

\section{Main results}

Theorem 2.1. Let $\left\{X_{n}, n \geq 1\right\}$ be a sequence of independent identically distributed random variables with cumulative distribution function $F(x)$ which is absolutely continuous with pdf $f(x)$ and $E\left(X^{k}\right)<\infty$. For positive integers $k, l$ and a positive real number $\theta<k$,

$$
(\theta-k)^{l} E\left[X_{U(n+l)}^{k} \mid X_{U(m)}=y\right]=\theta^{l} E\left[X_{U(n)}^{k} \mid X_{U(m)}=y\right], n \geq m+1
$$

if and only if

$$
F(x)=1-c x^{-\theta}, \quad 0<c \leq x, \quad \theta>0 .
$$

Proof. For necessary part, it is easy to see that (2.2) implies (2.1).

For the sufficiency part, using Ahsanullah formula(1995), we get the following equation

$$
\begin{aligned}
& \frac{(\theta-k)^{l}}{(n+l-m-1) !} \int_{y}^{\infty}\left(-\ln \frac{\bar{F}(x)}{\bar{F}(y)}\right)^{n+l-m-1} x^{k} f(x) d x \\
& =\frac{\theta^{l}}{(n-m-1) !} \int_{y}^{\infty}\left(-\ln \frac{\bar{F}(x)}{\bar{F}(y)}\right)^{n-m-1} x^{k} f(x) d x .
\end{aligned}
$$

Since $F(x)$ is absolutely continuous, we can differentiate $(n-m)$ times both sides of (2.3) with respect to $y$ and simplify to obtain the following equation

$$
\frac{1}{\bar{F}(y) \Gamma(l)} \int_{y}^{\infty}\left(-\ln \frac{\bar{F}(x)}{\bar{F}(y)}\right)^{l-1} x^{k} f(x) d x=\frac{\theta^{l}}{(\theta-k)^{l}} y^{k}
$$

By setting $s=\frac{\bar{F}(x)}{\bar{F}(y)}$ in $(2.4)$ we get

$$
\frac{1}{\Gamma(l)} \int_{0}^{1}\left[\bar{F}^{-1}(s \bar{F}(y))\right]^{k}(-\ln (s))^{l-1} d s=\frac{\theta^{l}}{(\theta-k)^{l}} y^{k} .
$$

Setting $\bar{F}(y)=t$, we find that

$$
\frac{1}{\Gamma(l)} \int_{0}^{1}\left[\bar{F}^{-1}(s t)\right]^{k}(-\ln (s))^{l-1} d s=\frac{\theta^{l}}{(\theta-k)^{l}}\left[\bar{F}^{-1}(t)\right]^{k}, \quad 0<t<1 .
$$


Dividing both sides by $\frac{(\theta-k)^{l}}{\theta^{l}}$ and substituting once again $s=e^{-v}$ and $t=e^{-w}$, we have

$$
\frac{(\theta-k)^{l}}{\theta^{l} \Gamma(l)} \int_{0}^{\infty}\left[\bar{F}^{-1}\left(e^{-(v+w)}\right)\right]^{k}(v)^{l-1} e^{-v} d v=\left[\bar{F}^{-1}\left(e^{-w}\right)\right]^{k}, \quad 0<w<\infty .
$$

Now we use applications of integrated Cauchy functional equation in C.R. Rao \& D.N. Shanbhag(1994) and let $H(w)=\left[\bar{F}^{-1}\left(e^{-w}\right)\right]^{k}$. Consequently,

$$
\int_{R^{+}} H(v+w) \mu(d v)=H(w), \quad 0<w<\infty,
$$

where $\mu$ is a finite measure on $R^{+}$, which is absolutely continuous with respect to the $[L]$ measure and is defined by

$$
\mu(d v)=\frac{(\theta-k)^{l}}{\theta^{l} \Gamma(l)}(v)^{l-1} e^{-v} d v .
$$

Observe that $H$ is strictly increasing on $[0, \infty)$ since it is a superposition of two strictly decreasing functions. Hence, since $H$ is continuous it follows that

$$
H(w)=\left\{\begin{array}{l}
\gamma+\alpha(1-\exp (\eta w)) \text { if } \eta \neq 0, \\
\gamma+\beta w \text { if } \eta=0, w>0,
\end{array}\right.
$$

where $\alpha, \beta, \gamma$ are all constants and $\eta$ is determined such that

$$
\int_{R^{+}} \exp (\eta x) \mu(d x)=1 .
$$

From (2.8) we get

$$
1=\frac{(\theta-k)^{l}}{\theta^{l} \Gamma(l)} \int_{0}^{\infty}(x)^{l-1} e^{(\eta-1) x} d x .
$$

which means that $\eta<1$, since the integral at the right-hand side has to converge. Consequently,

$$
\frac{(\theta-k)^{l}}{\theta^{l}}=(1-\eta)^{l} .
$$

From equation (2.10) we obtain $\eta=\frac{k}{\theta}$ and it follows that $0<\eta<1$. Using equation (2.7) we get

$$
\left[\bar{F}^{-1}\left(e^{-x}\right)\right]^{k}=\gamma+\alpha\left(1-e^{\eta x}\right) .
$$


By applying Theorem 1 in Anna Dembiǹska \& Jacek Wesolowski(2000) for $a=\frac{\theta^{l}}{(\theta-k)^{l}}>1$ and $\alpha+\gamma=0$, we have

$$
\bar{F}(z)=c z^{-\theta}, \quad 0<c \leq z, \quad \theta>0, \quad c=\gamma^{\frac{\theta}{k}} .
$$

Hence, the theorem is proved.

Theorem 2.2. Let $\left\{X_{n}, n \geq 1\right\}$ be a sequence of independent identically distributed random variables with cumulative distribution function $F(x)$ which is absolutely continuous with $p d f f(x)$. For positive integers $k, l$ and a positive real number $\theta$,

$$
E\left[X_{U(n+l)}^{k} \mid X_{U(m)}=y\right]=E\left[X_{U(n)}^{k} \mid X_{U(m)}=y\right]+l \theta^{k}, n \geq m+1 .
$$

if and only if

$$
F(x)=1-e^{-\left(\frac{x}{\theta}\right)^{k}}, 0 \leq x, \theta>0 .
$$

Proof. For necessary part, it is easy to see that (2.12) implies (2.11). For the sufficiency part, we get

$$
\begin{aligned}
& \frac{1}{(n+l-m-1) !} \int_{-\infty}^{y}\left(-\ln \frac{\bar{F}(x)}{\bar{F}(y)}\right)^{n+l-m-1} x^{k} f(x) d x \\
& =\frac{1}{(n-m-1) !} \int_{-\infty}^{y}\left(-\ln \frac{\bar{F}(x)}{\bar{F}(y)}\right)^{n-m-1} x^{k} f(x) d x+l \theta^{k} .
\end{aligned}
$$

Since $F(x)$ is absolutely continuous, we can differentiate $(n-m)$ times both sides of (2.13) with respect to $y$ and simplify to obtain the following equation

$$
\frac{1}{\bar{F}(y) \Gamma(l)} \int_{y}^{\infty}\left(-\ln \frac{\bar{F}(x)}{\bar{F}(y)}\right)^{l-1} x^{k} f(x) d x=y^{k}+l \theta^{k} .
$$

By setting $s=\frac{\bar{F}(x)}{\bar{F}(y)}$ in (2.14) we get

$$
\frac{1}{\Gamma(l)} \int_{0}^{1}\left[\bar{F}^{-1}(s \bar{F}(y))\right]^{k}(-\ln (s))^{l-1} d s=y^{k}+l \theta^{k} .
$$

Setting $\bar{F}(y)=t$, we find that

$$
\frac{1}{\Gamma(l)} \int_{0}^{1}\left[\bar{F}^{-1}(s t)\right]^{k}(-\ln (s))^{l-1} d s=\left[\bar{F}^{-1}(t)\right]^{k}+l \theta^{k}, \quad 0<t<1 .
$$

By setting $s=e^{-v}$ and $t=e^{-w}$, we obtain $\frac{1}{\Gamma(l)} \int_{0}^{\infty}\left[\bar{F}^{-1}\left(e^{-(v+w)}\right)\right]^{k}(v)^{l-1} e^{-v} d v=\left[\bar{F}^{-1}\left(e^{-w}\right)\right]^{k}+l \theta^{k}, 0<w<\infty$. 
In the same way as the proof of Theorem 1.1, we get

$$
1=\frac{1}{\Gamma(l)} \int_{0}^{\infty}(x)^{l-1} e^{(\eta-1) x} d x,
$$

which means that $\eta<1$, since the integral at the right-hand side has to converge. Consequently,

$$
1=(1-\eta)^{l}
$$

From equation (2.16) we obtain $\eta=0$.

Using equation (2.7) we get

$$
\left[\bar{F}^{-1}\left(e^{-x}\right)\right]^{k}=\gamma+\beta x .
$$

By applying Theorem 1 in Anna Dembiǹska \& Jacek Wesolowski(2000) for $\gamma=0, a=1$ and $\beta=\theta^{k}$, we have

$$
\bar{F}(z)=e^{-\left(\frac{z}{\theta}\right)^{k}}, \quad z \geq 0, \quad \theta>0 .
$$

Hence, the theorem is proved.

\section{References}

[1] M. Ahsanullah, Record Statistics, Inc, Dommack NY, 1995.

[2] M. Ahsanullah and M. Shakil, A note on the characterizations of the pareto distribution by upper record values, Commun. Korea Math. soc. 27 (2012), 835-842.

[3] A. Dembiǹska and J. Wesolowski, Linearity of regression for non-adjacent record values, J. Statist. Plann. Inference 90 (2000), 195-205.

[4] M. Y. Lee, Characterizations of the pareto distribution by conditional expectations of record values, Commun. Korea Math. soc. 18 (2003), 127-131.

[5] C. R. Rao and D. N. Shanbhag, Choquet-Deny Type Functional Equations with Applications to Stochastic Models, Wiley, NY, 1994.

Department of Mathematics

Dankook University

Cheonan 330-714, Republic of Korea

E-mail: hwjin@bistel.co.kr

$* *$

Department of Mathematics

Dankook University

Cheonan 330-714, Republic of Korea

E-mail: leemy@dankook.ac.kr 